\title{
Eye movements and the perception of rectilinear dot progressions
}

EDWARD A, HOLDEN, JR,

\section{E. R. JOHNSTONE TRAINING AND RESEARCH CENTER, BORDENTOWN, N. J.}

Three groups of high school students were tested on a task requiring "straight" or "crooked" judgments of rectilinear dot progressions generated by sequentially illuminated lights. Each group was tested under one of three conditions, which differed in the amount of eye movement occasioned by successive stimulus presentations. There were no significant differences between the numbers of "straight" judgments for the three groups. In all groups the number of "straight" judgments increased with faster presentation rates.

In a previous study (Holden, in press) in which Ss were required to judge whether rectilinear dot progressions appeared to form "straight" or "crooked" lines, it was found that a highly redundant series of light sequences (i.e., where each sequence started at the same point and progressed in the same direction) occasioned significantly more "straight" responses than did a less redundant series (i.e., where the sequences started at several different points and progressed in several different directions). On the assumption that the highly redundant series of light sequences effected closer proximity between anticipatory eye fixations and actual stimulus positions, it was speculated that the greater number of correct judgments might be attributable to the shorter eye movements necessary for successive stimulus fixations.

This study was undertaken to investigate the relationship between eye movements and perceptual veridicality in a task similar to that utilized for the low redundancy condition of the previous study (Holden, in press). It was hypothesized that veridical perception (in the present case "straight" judgments) of the spatial relationship between transient stimuli is inversely related to the amplitude of eye movement occasioned by successive stimulus presentations. Three groups of Ss were tested, each under a condition differing in the amplitude of eye movement between prestimulus fixation points and actual positions of the light stimuli. 1 It was predicted that the number of "straight" responses would be highest for the group with least eye movement, next highest for the group with intermediate movement, and lowest for the group with most movement.

\section{Subjects}

The Ss were 24 male and 24 female volunteers from a local high school. Eight males and eight females were assigned to each of the three experimental groups, mean CAs for which were, from least to most eye movement, $16.7,16.2$, and $16.5 \mathrm{yrs}$., respectively.

\section{Apparatus and Stimuli}

Essentials of the apparatus have been described in an earlier report (Holden, 1965). The task required Ss to judge the rectilinearity of geometrically straight dot progressions generated by sequentially illuminating four lights spatially distributed within a 4 by 4 matrix of neon lights (NE-2s). This matrix was covered with translucent black muslin, through which the unilluminated lamps could not be seen. The 10-dot progressions, each appearing as it would were all lights illuminated simultaneously, are shown by the open circles in Fig. 1. Arrows indicate the first light and direction of subsequent lamp illuminations for each sequence.

\section{Procedure}

Ss were tested individually in sessions requiring approximately $20 \mathrm{~min}$. A pretest was given at the beginning of each session to assure that Ss could discriminate between rectilinear and nonrectilinear sequences when the line stimuli were presented simultaneously as orange dots on black posterboard. The distributions of dots in the rectilinear pretest patterns were identical to those of lamps illuminated sequentially in the main experiment. To demonstrate the difference between straight and crooked lines, Ss were initially shown sequence No. 10 side by side with a sequence similar to No. 3 but in which one of the dots had been displaced $6 \mathrm{~mm}$ from rectilinearity. These two sequences, intermixed with the four different vertical and horizontal sequences in Fig. 1 (Nos. $1,2,6$, and 7) and their nonrectilinear homologues (one dot displaced $6 \mathrm{~mm}$ ) were then presented individually in random order for judgments of "straight" or "crooked." All Ss performed this pretest with no errors.

Ss were then instructed that they would see four dots of light go on and off one after the other on the pattern display screen, and that after each sequence they should tell $\mathrm{E}$ whether the four lights came on in a straight line or a crooked line. Before each sequence

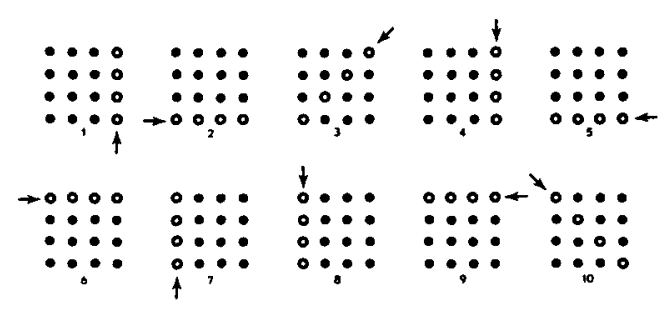

Fig. 1. Progressions of lamp illuminations in the 10 dot sequences. 


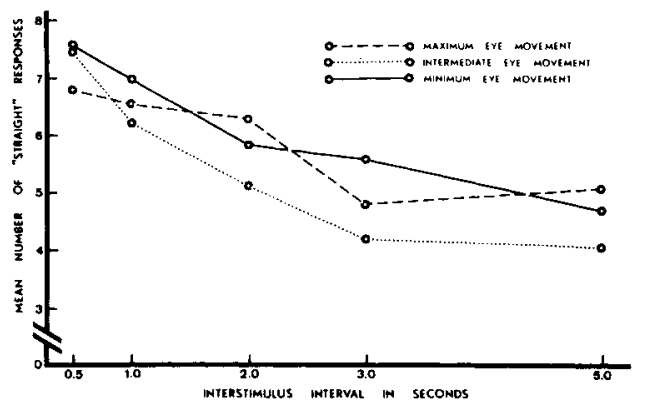

Fig. 2. Mean number of "straight" responses per interstimulus interval for each group.

E prepared S with a warning signal "Ready." All Ss received 50 trials, these consisting of all possible combinations of the 10 dot sequences with interstimulus intervals of $0.5,1.0,2.0,3.0$, and $5.0 \mathrm{sec}$. The duration of each light stimulus remained constant at $.05 \mathrm{sec}$. throughout the experiment. These combinations were randomized within two blocks of 25 trials each so that each sequence and interval occurred an equal number of times in each block. In each group four males and four females received Block 1 followed by Block 2, the remaining Ss receiving these blocks in reverse order.

Differential treatment of the three groups was as follows: (a) Minimum Movement Group: a small orange dot was placed on the display screen in the center of the light matrix, and Ss were instructed to fixate this point at the ready signal and to maintain fixation until the last light stimulus in each sequence. The vertical and horizontal distance between adjacentlights was 1 in. (b) Intermediate Movement Group: Ss had no fixation point. As in the preceding group, the vertical and horizontal distance between contiguous lights was 1 in. (c) Maximum Movement Group: The horizontal and vertical distance between contiguous lights was increased to $2 \mathrm{in}$., thus increasing the amount of eye movement necessary for fixating successive stimuli. As in the preceding group, Ss had no fixation point. Results and Discussion

Curves depicting the mean number of "straight" responses associated with each interstimulus interval in the three groups are plotted in Fig. 2. Statistical evaluation of these curves was made with a Lindquist (1953) Type I variance analysis, the results of which demonstrated a nonsignificant Group effect, a highly significant Interval effect $(F=30.76, d f=4 / 180, p<$ .001 ), and a nonsignificant interaction.

Failure to demonstrate differences between the three levels of eye movement is inconsistent with the hypothesis that veridical perception of the spatial relationship between transient stimuli is inversely related to the amplitude of eye movement occasioned by successive stimulus presentations. It thus appears that the larger number of "straight" responses obtained under the high redundancy condition of the previous study (Holden, in press) cannot be attributed to closer proximity between anticipatory eye fixations and actual stimulus loci as compared to the low redundancy condition. An alternative interpretation suggests that other factors deriving from repetition of the same sequence in the high redundancy condition were responsible. Possibly textural and other invariant stimuli associated with the pattern display screen were, by repeated and predictable association with the same light sequence on each trial, utilized as interstimulus mediation referents for the successive light positions. According to this interpretation, repetition of the same light sequence on every trial provided an association between each light stimulus and textural cues which served as spatial anchors during the interstimulus interval. This interpretation would, for future investigations, predict that enhancing textural cues should facilitate the number of "straight" responses.

The facilitation of "straight" responses with shorter interstimulus intervals is consistent with findings from the previous study (Holden, in press), thereby lending further support to the hypothesis that stimulus displacement (nonveridical perception, i.e., "crooked") reflects the dissipation of a locus defining trace, and that perceptual veridicality of the spatial relationship between immediately successive lights depends upon the degree of continuity between the traces of these lights.

\section{References}

Holden, E. A., Jr. Temporal factors and subnormality in visual pattern recognition: A test of stimulus trace theory. J. comp. physiol. Psychol, 1965, 59, 340-344.

Holden, E. A., Jr. Interstimulus interval, locus redundancy, and mental subnormality in the perception of rectilinear dot progressions. J. comp. physiol. Psychol., in press.

Lindquist, E. F. Design and analy sis of experiments in psychology and culcution. Boston: Houghton Mifflin Co., 1953.

\section{Note}

1. The group with an intermediate degree of eye movement was part of the preceding study. 